\title{
Identification of a novel autophagy-related gene signature for predicting metastasis and survival in patients with osteosarcoma
}

\section{Guangzhi Zhang}

Lanzhou University Second Hospital https://orcid.org/0000-0003-3193-0297

\section{Yajun Deng}

Lanzhou University Second Hospital

\section{Zuolong Wu}

Lanzhou University Second Hospital

\section{Enhui Ren}

Lanzhou University Second Hospital

\section{Wenhua Yuan}

Lanzhou University Second Hospital

\section{Qiqi Xie ( $\sim$ jieqq16@lzu.edu.cn)}

Lanzhou University Second Hospital https://orcid.org/0000-0003-4099-5287

\section{Primary research}

Keywords: osteosarcoma, autophagy-related genes, signature, survival, metastasis

Posted Date: March 26th, 2020

DOl: https://doi.org/10.21203/rs.3.rs-19384/v1

License: (1) This work is licensed under a Creative Commons Attribution 4.0 International License. Read Full License 


\section{Abstract}

Background: Osteosarcoma (OS) is a bone malignant tumor that occurs in children and adolescents. Due to a lack of reliable prognostic biomarkers, the prognosis of OS patients is often uncertain. This study aimed to construct an autophagy-related gene signature to predict the prognosis of OS patients.

Methods: The gene expression profile data of OS and normal muscle tissue samples were downloaded separately from the Therapeutically Applied Research To Generate Effective Treatments (TARGET) and Genotype-Tissue Expression (GTEx) databases. The differentially expressed autophagy-related genes (DEARGs) in OS and normal muscle tissue samples were screened using $\mathrm{R}$ software, before being subjected to Gene Ontology (GO) and Kyoto Encyclopedia of Genes and Genomes (KEGG) enrichment analysis. A protein-protein interaction (PPI) network was constructed and hub autophagy-related genes were screened. Finally, the screened autophagy-related genes were subjected to univariate Cox regression, Lasso Cox regression, survival analysis, and clinical correlation analysis.

Results: A total of 120 DEARGs and 10 hub autophagy-related genes were obtained. A prognostic autophagy-related gene signature consisting of 9 genes ( BNIP3 , MYC , BAG1 , CALCOCO2 , ATF4 , AMBRA1, EGFR, MAPK1, and PEX) was constructed. This signature was significantly correlated to the prognosis $(P<0.0001)$ and distant metastasis of OS patients $(P=0.013)$.

Conclusion: This signature based on 9 autophagy-related genes could predict metastasis and survival in patients with OS.

\section{Background}

Osteosarcoma (OS) is a common primary bone malignant tumor that occurs in children and adolescents, and has a high rate of metastasis and recurrence [1,2]. Approximately $10-20 \%$ of OS patients have numerous lesions associated with pain and swelling [3]. While many studies have shown that adjuvant chemotherapy could improve survival in patients with OS, the prognosis of OS patients remains poor, and the long-term survival rate is less than $20 \%[4,5]$. Improved understanding of disease pathogenesis and the identification of reliable prognostic biomarkers are crucial to better the prognosis of patients with OS.

Autophagy is a highly conserved intracellular degradation pathway that maintains homeostasis by degrading intracellular macromolecules and organelles through the formation of autophagosomes and subsequent fusion with lysosomes [6, 7]. Autophagy is widely involved in the physiological and pathological processes of cells in vivo, such as cell homeostasis, hematopoiesis, organ development, neurodegeneration, and tumorigenesis [8-10]. Basal levels of autophagy are employed by cells under normal circumstances. When cells become deficient in nutrition or are exogenously stimulated, their autophagy levels increase accordingly to cope with unfavorable factors, as an adaptive response to metabolic stress [10]. Many studies have shown that autophagy could promote or inhibit the survival of tumor cells at different stages of cancer development [11]. In the early stages of cancer, autophagy promotes the degradation of damaged proteins and organelles to reduce cellular damage and 
chromosomal instability, thereby inhibiting cancer progression. However, once cancer has formed, autophagy allows tumor cells to survive under stressful conditions, thereby promoting tumor progression [12]. The relationship between autophagy and OS has been previously reported. For example, in a mouse OS model, silencing BECN1 (an autophagy-promoting gene) could increase OS cell metastasis [13]. By regulating autophagy in human OS MG-63 cells, the survival rate of OS cells and cisplatin-induced chemoresistance was significantly increased [14]. These studies have confirmed that autophagy is closely related to OS, suggesting that autophagy-related genes may have potential application as prognostic markers of OS.

However, autophagy is a complex process involving hundreds of molecules. Therefore, models that integrate multiple autophagy-related genes can improve the accuracy of prognostic prediction[15]. Compared to traditional single-molecule prognosis predictors, genomic profiling based on "Omics" could predict the prognosis of a set of genes for patients, which are called "classifiers" or "signatures"[16]. With the rapid development of gene expression profile chip technology and bioinformatics methods, it has become feasible to use high-throughput expression profile data to analyze the relationship between autophagy-related gene expression and the clinical outcomes of cancer patients [17]. Therefore, in this study, we used a bioinformatics approach to identify potential autophagy-related genes for use as predictive biomarkers of the prognosis of OS patients.

\section{Materials And Methods}

\subsection{Data retrieval and processing}

Therapeutically Applicable Research To Generate Effective Treatments (TARGET, https://ocg.cancer.gov/programs/target) is an open database for childhood cancers. It seeks to use a comprehensive genomic approach to identify molecular changes in the occurrence and development of childhood cancers that are difficult to treat. The Genotype-Tissue Expression (GTEx, https://www.gtexportal.org/home/) database provides transcriptome data of various normal human tissues [18]. We downloaded the gene expression profile data set and clinical information of OS patients from the TARGET database, which included 88 OS samples, and downloaded the gene expression profile data set of the control group from the GTEx database, which included 396 normal muscle tissue samples. The sva package [19] was used to remove the differences between different batches in the expression matrix of the two datasets, and then merge them for subsequent analysis.

\subsection{Identification of differentially expressed autophagy-related genes (DEARGs)}

The Human Autophagy Database (HADb, http://autophagy.lu/clustering/index.html) is the first public database dedicated to human autophagy, which contains a complete and current list of human autophagy-related genes reported in literature from PubMed and biological public databases[20]. The 222 autophagy genes were obtained from the HADb. The limma package [21] was used to screen DEARGs between OS samples and normal muscle tissue samples. The intercept value was Adjusted P (adj. P) values $<0.05$. 


\subsection{DEARGs function and pathway enrichment analysis}

Gene ontology (GO) is an important method and tool for annotating genes and their products, which is beneficial for the integration and utilization of biological data [22]. Kyoto Encyclopedia of Genes and Genomes (KEGG) is a database integrating genomics, chemistry, and system function information, which can provide currently known biological metabolic signaling pathways [23]. ClusterProfiler package [24] was used to perform GO and KEGG enrichment analysis on DEARGs. FDR $<0.05$ was set as the threshold for significant gene enrichment.

\subsection{PPI network construction, hub autophagy-related gene selection, and functional similarity analysis of DEARGs}

STRING (http://www.string-db.org/, Version: 11.0) is a biological tool for evaluating PPI information [25]. The DEARGs were imported into the STRING online software, and the PPI network was derived, and exported in TSV format. The interactions with a combined score of greater than 0.9 were considered statistically significant. The obtained source file was then imported into Cytoscape software [26], and the cytoHubba plug-in [27] was used to screen the top 10 hub autophagy-related genes according to the maximum correlation standard algorithm. Based on the semantic similarity of gene annotation $\mathrm{GO}$ terms [28], we applied the GOSemSim package [29] to calculate the strength of the relationship between molecular function and cell localization among the 10 hub genes, and the average value of functional similarity to compare to the 10 hub autophagy-related genes were sorted with a cut-off value of 0.65 , and the results were visualized using the ggplot2 package [24].

\subsection{Construction of prognostic autophagy-related gene signature}

First, we performed univariate Cox regression analysis on the obtained DEARGs to screen for genes with prognostic value; the screening criterion was $P<0.05$. Then, glmnet software [30] was used to perform Lasso Cox regression analysis on genes with prognostic value. Based on the gene expression level, an prognostic autophagy-related gene signature for OS patients was constructed. A prognostic risk score for each sample based on this signature was derived, and patients were divided into high and low-risk groups based on the median value of the risk score. The risk factor correlation diagram was drawn using $\mathrm{R}$ language to show the prognosis of patients in the high and low-risk groups. The difference in survival between the high and low-risk groups was evaluated by Kaplan-Meier curve.

\subsection{Relationship between prognostic autophagy-related gene signature and clinical characteristics}

To further evaluate the correlation between prognostic autophagy-related gene signatures and clinical characteristics, we used the ggstatsplot package (https://github.com/IndrajeetPatil/ggstatsplot) to perform correlation analysis between the obtained risk scores and clinical data (age, gender, and metastasis) from the TARGET database, and the results are shown graphically through the ggplot2 package (CRAN.R-project.org/package = ggplot2). 


\section{Results}

\subsection{Data processing and identification of DEARGs}

After the obtained data were subjected to background correction, data normalization, and removal of the difference between batches, a total of 222 autophagy-related genes and 120 DEARGs were obtained.

\subsection{DEARGs function and pathway enrichment analysis}

The results of GO analysis demonstrated that the biological process (BP) changes of the DEARGs were significantly enriched in 'autophagy', 'process utilizing autophagic mechanism', 'macroautophagy', 'autophagosome assembly', 'autophagosome organization', 'regulation of autophagy', 'vacuole organization', and 'autophagy of mitochondrion'; Cell components (CC) were mainly enriched in 'autophagosome', 'phagophore assembly site', 'autophagosome membrane', 'vacuolar membrane', 'melanosome', 'pigment granule', 'phagophore assembly site membrane', and 'late endosome'; Molecular function (MF) mainly involved 'ubiquitin protein ligase binding', 'ubiquitin-like protein ligase binding', 'chaperone binding', 'protein serine/threonine kinase activity', and 'heat shock protein binding'. KEGG pathway enrichment was mainly enriched in 'autophagy', 'shigellosis', 'kaposi sarcoma-associated herpesvirus infection', 'human cytomegalovirus infection', 'mitophagy', 'protein processing in endoplasmic reticulum', and 'apoptosis' (Figure 1A).

\subsection{PPI network construction, hub autophagy-related gene selection, and functional similarity analysis of DEARGs}

The results of the PPI network showed that three genes, including TP53, ATG16, and MTOR, had larger weights and stronger correlations (Figure 2A). The interaction maps of the 10 hub autophagy-related genes (BECN1, ATG7, PIK3C3, ATG12, GABARAPL1, GABARAPL2, SQSTM1, MAP1LC3A, MAP1LC3B, and GABARAP) were screened (Figure 2B and Table 1).

To further identify the strength of the interactions between the hub autophagy-related genes, we sorted them based on their average functional similarity, and the results showed that MAP1LC3B, GABARAPL2, GABARAPL1, and GABARAP were the genes with the closest interactions. Among them, MAP1LC3B is the only gene with a cut-off value $>0.7$, with the highest average functional similarity (Figure $2 \mathrm{C}$ ).

\subsection{Construction of prognostic autophagy-related genes signature}

To study the prognostic value of DEARGs, the univariate Cox analysis revealed that 25 genes including BNIP3, MYC, VEGFA, ATG9B, NKX2.3, ATG4A, BAG1, CALCOCO2, ATF4, ATG7, ATG4D, AMBRA1, BAK1, APOL 1, EGFR, CCL2, MAPK1, CASP1, MAP2K7, DLC1, DNAJB9, VAMP3, PEX3, CTSD, and BCL2 had a prognostic value for OS (Figure 3 and Supplementary material 1). To further improve the accuracy of the results, we performed Lasso Cox regression analysis on the 25 DEARGs. The results showed that 9 genes (BNIP3, MYC, BAG1, CALCOCO2, ATF4, AMBRA1, EGFR, MAPK1, and PEX3) were correlated to prognosis (Figure 4 and Table 2). These 9 genes were subsequently used to construct a gene signature. We 
calculated the risk score of each sample, and divided the samples into high-risk and low-risk groups using the median risk as a cut-off value, and obtained a risk factor correlation diagram of the prognostic autophagy-related gene risk score. Compared to the low-risk group, the number of deaths in the high-risk group was significantly greater, and the levels of ATF4, BAG1, MYC, and BNIP3 were higher in the high-risk group, while the expression levels of MAPK1, EGFR, CALCOCO2, and AMBRA1 were lower (Figure 5A). Kaplan-Meier survival analysis showed that the overall survival rate of patients in the high-risk group was significantly lower than that in the low-risk group $(P<0.0001)$ (Figure 5B).

\subsection{Relationship between prognostic autophagy-related gene signature and clinical characteristics}

As shown in Figure 6 and Supplementary material 2, the risk score was significantly associated with the distant metastasis of OS $(P=0.05)$, but not with age $(P=0.710)$ and gender $(P=0.887)$.

\section{Discussion}

OS is the most common bone malignancy in children and adolescents, and is also the leading cause of disability and death in this age-group [31]. Although adjuvant chemotherapy can improve patient prognosis, the long-term survival rate of patients after relapse remains less than $20 \%$ [32]. Many studies have shown that autophagy-related proteins are closely related to the prognosis of patients with OS. Low expression of AGT5 may be related to the poor prognosis of OS patients [33]. Silencing the autophagypromoting gene BECN1 will increase cancer cell metastasis [13]. Currently, hundreds of proteins are thought to be involved in the autophagy process. In view of the importance of autophagy in OS, it can be reasonably speculated that autophagy-related genes have broad prospects in predicting prognosis, and multi-gene signatures generated by reliable algorithms will be superior to single molecules in predicting the prognosis of OS patients. In this study, for the first time, we integrated the genetic chip sequencing data of OS samples from the TARGET and GTEx databases with normal muscle tissue samples through bioinformatics methods to construct a prognostic autophagy-related gene signature for identifying prognostic OS biomarkers.

A total of 120 DEARGs were screened in this study. GO results showed that the BP of DEARGs mainly involved autophagy, macroautophagy, autophagosome assembly, autophagosome organization, and autophagy of mitochondrion; the $\mathrm{CC}$ were mainly enriched in autophagosome, phagophore assembly site, autophagosome membrane, vacuolar membrane, and late endosome; and the MF mainly involves ubiquitin protein ligase binding, chaperone binding, protein serine/ threonine kinase activity, and heat shock protein binding. Previous studies have shown that autophagy [33], macroautophagy [10], autophagosome [34], chaperone binding [35], threonine kinase activity [36], and heat shock protein binding [37] are closely related to the occurrence, invasion, and transfer of OS. KEGG pathway enrichment analysis showed that the enriched pathway mainly involved autophagy, mitophagy, and apoptosis. Studies have shown that autophagy plays an important role in the proliferation, invasion, metastasis, apoptosis, and drug resistance of OS [38,39]. The role of mitophagy has been widely studied and is considered to be an early manifestation of cellular autophagy; mitochondrial function is impaired after 
mitochondrial damage and induces mitophagy [40]. Mei et al. [41] showed that mitophagy is involved in the proliferation and apoptosis of OS cell lines. Parunya et al. [42] showed that endoplasmic reticulum protein 29 (ERp29) expression was significantly higher in OS cells and was negatively correlated to patient survival time. All these findings are consistent with the data mining results of the present study.

BECN1, ATG7, ATG12, PIK3C3, GABARAP, GABARAPL1, GABARAPL2, MAP1LC3A, MAP1LC3B, and SQSTM1 are the hub autophagy-related genes identified in this study. BECN1 affects the drug sensitivity of OS cells by mediating the autophagy process [43]. ATG12 is involved in osteosarcoma cell metastasis [44], and ATG7 is associated with the invasiveness of bladder cancer cells [45]. The expression level of PIK3C3 in breast cancer gradually increases with disease progression [46], and low expression of GABARAPL1 is associated with poor prognosis in patients with breast cancer and hepatocellular carcinoma $[47,48]$. The accumulation of MAP1LC3A in the nucleus of cancer cells is closely related to the poor prognosis of non-small cell lung cancer [49], and the low expression of MAP1LC3B is related to the poor prognosis of breast cancer [50]. High SQSTM1 expression is associated with poor prognosis in human acute lymphoblastic leukemia [51]. Taken together, the above studies support our analysis, but the role of these genes in OS is unclear. Considering the similarity of hub autophagy-related genes in CC and MF, we sorted by semantic similarity and found that MAP1LC3B, GABARAPL2, GABARAPL1, and GABARAP are the genes with the closest interaction. As members of the GABARAP family, GABARAP, GABARAPL1, and GABARAPL2 are widely involved in intracellular transport and autophagy pathways [48]. This further illustrates the reliability of the analysis results of this study.

Univariate Cox regression and Lasso Cox regression analysis showed that a gene signature composed of 9 autophagy-related genes (BNIP3, MYC, BAG1, CALCOCO2, ATF4, AMBRA1, EGFR, MAPK1, and PEX3) was closely related to the prognosis of OS. Studies have shown that the abnormal expression of MYC [52], ATF4 [53], and MAPK1 [54] is related to the proliferation, migration, and invasion of OS cells. As a transcription target of MYC, BAG1 cooperates with HSP70 molecular chaperone to selectively mediate MYC overexpression and affect the survival of OS cells [55]. The abnormal expression of BNIP3 is associated with the prognosis of renal cancer [56], breast cancer [57], and salivary adenoid cystic carcinoma [16]. Abnormal expression of AMBRA1 is associated with prognosis of bile duct cancer and cutaneous melanoma $[58,59]$. Xiang et al. [60] showed that abnormal expression of EGFR is related to drug resistance in tumor patients. Chu et al. [61] showed that PEX3 is related to the invasion and metastasis of ovarian cancer cells. Importantly, our results show that the prognostic autophagy-related gene signature is significantly associated with OS metastasis, but not with age and gender. The autophagy-related gene signature revealed in this study are closely related to the clinical prognosis of OS patients, which may have important significance for the prognostic management and monitoring of these patients.

Current research on the prognosis of OS is mainly based on the prognosis of single genes, and the signature of multiple genes could well avoid the differences caused by individual heterogeneity. We used univariate Cox analysis, Lasso Cox, and survival analysis to predict the prognosis of OS patients by including multiple genes as a whole, and further verified by clinical correlation analysis. Therefore, this 
prognostic model has high prognostic value. However, our research has certain limitations. We used the recently opened TARGET database. Although it contains relatively complete clinical information of OS patients, this database lacks control group sample data. Therefore, the number of control group samples used in this study was derived from the GTEx database, which may lead to the data having a certain heterogeneity. In addition, there is currently an insufficient dataset to further verify the accuracy of our analysis results. Therefore, large-scale analysis of clinical tissue specimens is required in the future.

\section{Conclusion}

In conclusion, through in-depth analysis of OS at the level of autophagy genes, we constructed a novel prognostic autophagy-related gene signature based on 9 genes (BNIP3, MYC, BAG1, ALCOCO2, ATF4, AMBRA1, EGFR, MAPK1, and PEX3), which was used to predict the metastasis and survival of OS patients. Our finding suggests that the 9 prognostic autophagy-related gene signature may help facilitate personalized medicine in the clinical setting.

\section{Abbreviations}

OS: Osteosarcoma; GTEx: Genotype-Tissue Expression; TARGET: Therapeutically Applied Research To Generate Effective Treatments; GO: Gene Ontology; KEGG: Kyoto Encyclopedia of Genes and Genomes; PPI: protein-protein interaction; DEARGs: differentially expressed autophagy-related genes; HADb: Human Autophagy Database; BP: biological process; CC: cell components; MF: molecular function ; HR: hazard ratio; Lasso: least absolute shrinkage and selection operator.

\section{Declarations}

\section{Ethics approval and consent to participate}

Not applicable.

\section{Consent for publication}

All authors agreed on the manuscript

\section{Availability of data and materials}

The datasets used and/or analyzed during the current study are available from the corresponding author upon reasonable request.

\section{Competing interests}

The authors declare that they have no competing interests.

\section{Funding}


No funding was received.

Authors' contributions

GZZ, YJD, ZLW and QQX conceived and designed the study. EHR and WHY contributed to dataset selection and bioinformatics analysis. GZZ, YJD and QQX wrote and revised the manuscript. All authors read and approved the final manuscript.

\section{Acknowledgements}

This work has benefited from TARGET and GTEx. We thank the TARGET and GTEx network for its generous sharing large amounts of data. We would like to thank FigureYa (Xiao Ya Hua Tu) for the figure technology support. We would like to thank Editage (www.editage.cn) for English language editing.

\section{Authors' information}

${ }^{1}$ The Second Clinical Medical College, Lanzhou University, Lanzhou, Gansu 730000, PR China;

${ }^{2}$ Department of Orthopedics, Lanzhou University Second Hospital, Lanzhou, Gansu 730000, PR China;

${ }^{3}$ Lin Tao County Hospital of Traditional Chinese Medicine, Dingxi, Gansu 730050, PR China.

\section{References}

1. Rickel K, Fang F, Tao J: Molecular genetics of osteosarcoma. BONE 2017, 102:69-79.

2. Wedekind MF, Wagner LM, Cripe TP: Immunotherapy for osteosarcoma: Where do we go from here? PEDIATR BLOOD CANCER 2018, 65(9):e27227.

3. Liu Y, Li W, Chang F, Liu J, Lin J, Chen D: MicroRNA-505 is downregulated in human osteosarcoma and regulates cell proliferation, migration and invasion. ONCOL REP 2018, 39(2):491-500.

4. Tian X, He Y, Han Z, Su H, Chu C: The Cytoplasmic Expression Of CLDN12 Predicts An Unfavorable Prognosis And Promotes Proliferation And Migration Of Osteosarcoma. CANCER MANAG RES 2019, 11:9339-9351.

5. Wang J, Liu S, Shi J, Li J, Wang S, Liu H, Zhao S, Duan K, Pan X, Yi Z: The Role of miRNA in the Diagnosis, Prognosis, and Treatment of Osteosarcoma. Cancer biotherapy \& radiopharmaceuticals 2019, 34(10):605-613.

6. Folkerts $\mathrm{H}$, Hilgendorf $\mathrm{S}$, Vellenga $\mathrm{E}$, Bremer $\mathrm{E}$, Wiersma VR: The multifaceted role of autophagy in cancer and the microenvironment. MED RES REV 2019, 39(2):517-560.

7. Escobar KA, Cole NH, Mermier CM, VanDusseldorp TA: Autophagy and aging: Maintaining the proteome through exercise and caloric restriction. AGING CELL 2019, 18(1):e12876.

8. Sotthibundhu A, Promjuntuek W, Liu M, Shen S, Noisa P: Roles of autophagy in controlling stem cell identity: a perspective of self-renewal and differentiation. CELL TISSUE RES 2018, 374(2):205-216.

9. Menzies FM, Fleming A, Caricasole A, Bento CF, Andrews SP, Ashkenazi A, Füllgrabe J, Jackson A, Jimenez Sanchez M, Karabiyik C et al: Autophagy and Neurodegeneration: Pathogenic Mechanisms 
and Therapeutic Opportunities. NEURON 2017, 93(5):1015-1034.

10. Wang L, Ye X, Zhao T: The physiological roles of autophagy in the mammalian life cycle. Biological reviews of the Cambridge Philosophical Society 2019, 94(2):503-516.

11. An Y, Bi F, You Y, Liu X, Yang Q: Development of a Novel Autophagy-related Prognostic Signature for Serous Ovarian Cancer. J CANCER 2018, 9(21):4058-4071.

12. Levy JMM, Towers CG, Thorburn A: Targeting autophagy in cancer. Nature reviews. Cancer 2017, 17(9):528-542.

13. Zhang F, Yan T, Guo W, Sun K, Wang S, Bao X, Liu K, Zheng B, Zhang H, Ren T: Novel oncogene COPS3 interacts with Beclin1 and Raf-1 to regulate metastasis of osteosarcoma through autophagy. Journal of experimental \& clinical cancer research : CR 2018, 37(1):135.

14. Kim M, Jung J, Choi S, Lee H, Morales LD, Koh J, Kim SH, Choi Y, Choi C, Slaga TJ et al: GFRA1 promotes cisplatin-induced chemoresistance in osteosarcoma by inducing autophagy. AUTOPHAGY 2017, 13(1):149-168.

15. Liu F, Xing L, Zhang X, Zhang X: A Four-Pseudogene Classifier Identified by Machine Learning Serves as a Novel Prognostic Marker for Survival of Osteosarcoma. GENES-BASEL 2019.

16. Zhanwei C, Dubin S, Shengyun H, Haiwei W, Dongsheng Z: Analysis of BNIP3 expression and clinical research in salivary adenoid cystic carcinoma. Hua xi kou qiang yi xue za zhi = Huaxi kouqiang yixue zazhi $=$ West China journal of stomatology 2016, 34(4):404-407.

17. Yang X, Zhu S, Li L, Zhang L, Xian S, Wang Y, Cheng Y: Identification of differentially expressed genes and signaling pathways in ovarian cancer by integrated bioinformatics analysis. ONCOTARGETS THER 2018, 11:1457-1474.

18. GTEx C: Human genomics. The Genotype-Tissue Expression (GTEx) pilot analysis: multitissue gene regulation in humans. Science (New York, N.Y.) 2015, 348(6235):648-660.

19. Parker HS, Leek JT, Favorov AV, Considine M, Xia X, Chavan S, Chung CH, Fertig EJ: Preserving biological heterogeneity with a permuted surrogate variable analysis for genomics batch correction. Bioinformatics (Oxford, England) 2014, 30(19):2757-2763.

20. Moussay E, Kaoma T, Baginska J, Muller A, Van Moer K, Nicot N, Nazarov PV, Vallar L, Chouaib S, Berchem $\mathrm{G}$ et al: The acquisition of resistance to TNFa in breast cancer cells is associated with constitutive activation of autophagy as revealed by a transcriptome analysis using a custom microarray. AUTOPHAGY 2011.

21. Ritchie ME, Phipson B, Wu D, Hu Y, Law CW, Shi W, Smyth GK: limma powers differential expression analyses for RNA-sequencing and microarray studies. NUCLEIC ACIDS RES 2015, 43(7):e47.

22. Harris MA, Clark J, Ireland A, Lomax J, Ashburner M, Foulger R, Eilbeck K, Lewis S, Marshall B, Mungall $C$ et al: The Gene Ontology (GO) database and informatics resource. NUCLEIC ACIDS RES 2004, 32(Database issue):D258-D261.

23. Kanehisa M, Furumichi M, Tanabe M, Sato Y, Morishima K: KEGG: new perspectives on genomes, pathways, diseases and drugs. NUCLEIC ACIDS RES 2017, 45(D1):D353-D361. 
24. Yu G, Wang LG, Han Y, He QY: clusterProfiler: an R package for comparing biological themes among gene clusters. OMICS 2012, 16(5):284-287.

25. Szklarczyk D, Gable AL, Lyon D, Junge A, Wyder S, Huerta-Cepas J, Simonovic M, Doncheva NT, Morris JH, Bork P et al: STRING v11: protein-protein association networks with increased coverage, supporting functional discovery in genome-wide experimental datasets. NUCLEIC ACIDS RES 2019, 47(D1):D607-D613.

26. Shannon P, Markiel A, Ozier O, Baliga NS, Wang JT, Ramage D, Amin N, Schwikowski B, Ideker T: Cytoscape: a software environment for integrated models of biomolecular interaction networks. GENOME RES2003, 13(11):2498-2504.

27. Chin $\mathrm{CH}$, Chen $\mathrm{SH}, \mathrm{Wu} \mathrm{HH}, \mathrm{Ho} \mathrm{CW}$, Ko MT, Lin CY: cytoHubba: identifying hub objects and subnetworks from complex interactome. BMC SYST BIOL 2014, 8 Suppl 4:S11.

28. Sevilla JL, Segura V, Podhorski A, Guruceaga E, Mato JM, Martínez-Cruz LA, Corrales FJ, Rubio A: Correlation between gene expression and $\mathrm{GO}$ semantic similarity. IEEE/ACM transactions on computational biology and bioinformatics 2005, 2(4):330-338.

29. Yu G, Li F, Qin Y, Bo X, Wu Y, Wang S: GoSemSim: an R package for measuring semantic similarity among GO terms and gene products. Bioinformatics (Oxford, England) 2010, 26(7):976-978.

30. Friedman J, Hastie T, Tibshirani R: Regularization Paths for Generalized Linear Models via Coordinate Descent. J STAT SOFTW 2010.

31. Xiao H, Jensen PE, Chen X: Elimination of Osteosarcoma by Necroptosis with Graphene OxideAssociated Anti-HER2 Antibodies. INT J MOL SCI2019, 20(18):4360.

32. Nugent M: microRNA and Bone Cancer. ADV EXP MED BIOL 2015, 889:201-230.

33. Zhao G, Gao Z, Zhang Q, Tang X, Lv Y, Zhang Z, Zhang Y, Tan Q, Peng D, Jiang D et al: TSSC3 promotes autophagy via inactivating the Src-mediated PI3K/Akt/mTOR pathway to suppress tumorigenesis and metastasis in osteosarcoma, and predicts a favorable prognosis. Journal of experimental \& clinical cancer research : CR 2018.

34. Zhu J, Yu W, Liu B, Wang Y, Shao J, Wang J, Xia K, Liang C, Fang W, Zhou C et al: Escin induces caspase-dependent apoptosis and autophagy through the ROS/p38 MAPK signalling pathway in human osteosarcoma cells in vitro and in vivo. CELL DEATH DIS 2017, 8(10):e3113.

35. Chaiyawat P, Sungngam P, Teeyakasem P, Sirikaew N, Klangjorhor J, Settakorn J, Diskul-NaAyudthaya P, Chokchaichamnankit D, Srisomsap C, Svasti J et al: Protein profiling of osteosarcoma tissue and soft callus unveils activation of the unfolded protein response pathway. INT J ONCOL 2019, 54(5):1704-1718.

36. Wu Z, Liu J, Hu S, Zhu Y, Li S: Serine/Threonine Kinase 35, a Target Gene of STAT3, Regulates the Proliferation and Apoptosis of Osteosarcoma Cells. Cellular physiology and biochemistry : international journal of experimental cellular physiology, biochemistry, and pharmacology 2018, 45(2):808-818.

37. Yang J, Li Y, He M, Qiao J, Sang Y, Cheang LH, Gomes FC, Hu Y, Li Z, Liu N et al: HSP90 regulates osteosarcoma cell apoptosis by targeting the p53/TCF-1-mediated transcriptional network. J CELL 
PHYSIOL 2020, 235(4):3894-3904.

38. Liu K, Hou Y, Liu Y, Zheng J: LncRNA SNHG15 contributes to proliferation, invasion and autophagy in osteosarcoma cells by sponging miR-141. J BIOMED SCI 2017, 24(1):46.

39. Meng Y, Gao R, Ma J, Zhao J, Xu E, Wang C, Zhou X: MicroRNA-140-5p regulates osteosarcoma chemoresistance by targeting HMGN5 and autophagy. SCI REP-UK 2017, 7(1):416.

40. Melser S, Lavie J, Bénard G: Mitochondrial degradation and energy metabolism. Biochimica et biophysica acta 2015, 1853(10 Pt B):2812-2821.

41. Mei L, Sang W, Cui K, Zhang Y, Chen F, Li X: Norcantharidin inhibits proliferation and promotes apoptosis via c-Met/Akt/mTOR pathway in human osteosarcoma cells. CANCER SCI2019, 110(2):582-595.

42. Chaiyawat P, Pruksakorn D, Pipatwattana P, Phanphaisarn A, Teeyakasem P, Klangjorhor J, Settakorn $\mathrm{J}$ : Endoplasmic reticulum protein 29 (ERp29) as a novel prognostic marker and tumor suppressor in osteosarcoma. J BONE ONCOL 2019, 16:100233.

43. Huang J, Liu K, Yu Y, Xie M, Kang R, Vernon P, Cao L, Tang D, Ni J: Targeting HMGB1-mediated autophagy as a novel therapeutic strategy for osteosarcoma. AUTOPHAGY 2012, 8(2):275-277.

44. Bao X, Zhao L, Guan H, Li F: Inhibition of LCMR1 and ATG12 by demethylation-activated miR-570-3p is involved in the anti-metastasis effects of metformin on human osteosarcoma. CELL DEATH DIS 2018, 9(6):611.

45. Zhu J, Tian Z, Li Y, Hua X, Zhang D, Li J, Jin H, Xu J, Chen W, Niu B et al: ATG7 Promotes Bladder Cancer Invasion via Autophagy-Mediated Increased ARHGDIB mRNA Stability. Advanced science (Weinheim, Baden-Wurttemberg, Germany) 2019, 6(8):1801927.

46. Ling B, Chen L, Liu Q, Yang J: Gene expression correlation for cancer diagnosis: a pilot study. BIOMED RES INT 2014, 2014:253804.

47. Liu C, Xia Y, Jiang W, Liu Y, Yu L: Low expression of GABARAPL1 is associated with a poor outcome for patients with hepatocellular carcinoma. ONCOL REP 2014, 31(5):2043-2048.

48. Hervouet E, Claude-Taupin A, Gauthier T, Perez V, Fraichard A, Adami P, Despouy G, Monnien F, Algros $M$, Jouvenot $M$ et al: The autophagy GABARAPL1 gene is epigenetically regulated in breast cancer models. BMC CANCER 2015, 15:729.

49. Karpathiou G, Sivridis E, Koukourakis MI, Mikroulis D, Bouros D, Froudarakis ME, Giatromanolaki A: Light-chain $3 \mathrm{~A}$ autophagic activity and prognostic significance in non-small cell lung carcinomas. CHEST 2011, 140(1):127-134.

50. Ladoire S, Penault-Llorca F, Senovilla L, Dalban C, Enot D, Locher C, Prada N, Poirier-Colame V, Chaba $\mathrm{K}$, Arnould $\mathrm{L}$ et al: Combined evaluation of LC3B puncta and HMGB1 expression predicts residual risk of relapse after adjuvant chemotherapy in breast cancer. AUTOPHAGY 2015, 11(10):1878-1890.

51. Nguyen TD, Shaid S, Vakhrusheva O, Koschade SE, Klann K, Thölken M, Baker F, Zhang J, Oellerich T, Sürün $D$ et al: Loss of the selective autophagy receptor p62 impairs murine myeloid leukemia progression and mitophagy. BLOOD 2019, 133(2):168-179. 
52. Chen D, Zhao Z, Huang Z, Chen D, Zhu X, Wang Y, Yan Y, Tang S, Madhavan S, Ni W et al: Super enhancer inhibitors suppress MYC driven transcriptional amplification and tumor progression in osteosarcoma. BONE RES 2018, 6:11.

53. Zeng H, Zhang J, Du Y, Wang J, Ren Y, Li M, Li H, Cai Z, Chu Q, Yang C: Crosstalk between ATF4 and MTA1/HDAC1 promotes osteosarcoma progression. Oncotarget 2016, 7(6):7329-7342.

54. Wu J, Zhang C, Chen L: MiR-511 mimic transfection inhibits the proliferation, invasion of osteosarcoma cells and reduces metastatic osteosarcoma tumor burden in nude mice via targeting MAPK1. Cancer biomarkers : section A of Disease markers 2019, 26(3):343-351.

55. Gennaro VJ, Wedegaertner H, McMahon SB: Interaction between the BAG1S isoform and HSP70 mediates the stability of anti-apoptotic proteins and the survival of osteosarcoma cells expressing oncogenic MYC. BMC CANCER 2019, 19(1):258.

56. Macher-Goeppinger S, Keith M, Hatiboglu G, Hohenfellner M, Schirmacher P, Roth W, Tagscherer KE: Expression and Functional Characterization of the BNIP3 Protein in Renal Cell Carcinomas. TRANSL ONCOL 2017, 10(6):869-875.

57. Schulten H, Bangash M, Karim S, Dallol A, Hussein D, Merdad A, Al-Thoubaity FK, Al-Maghrabi J, Jamal A, Al-Ghamdi $\mathrm{F}$ et al: Comprehensive molecular biomarker identification in breast cancer brain metastases. J TRANSL MED 2017, 15(1):269.

58. Nitta T, Sato Y, Ren XS, Harada K, Sasaki M, Hirano S, Nakanuma Y: Autophagy may promote carcinoma cell invasion and correlate with poor prognosis in cholangiocarcinoma. INT J CLIN EXP PATHO 2014, 7(8):4913-4921.

59. Tang DYL, Ellis RA, Lovat PE: Prognostic Impact of Autophagy Biomarkers for Cutaneous Melanoma. FRONT ONCOL 2016, 6:236.

60. Ji XL, He M: Sodium cantharidate targets STAT3 and abrogates EGFR inhibitor resistance in osteosarcoma. Aging 2019, 11(15):5848-5863.

61. Chu $P, X u L, S u H:$ HULC functions as an oncogene in ovarian carcinoma cells by negatively modulating miR-125a-3p. J PHYSIOL BIOCHEM 2019, 75(2):163-171.

\section{Tables}


Table 1. Functions and scores of $10 \mathrm{hub}$ autophagy-related genes

\begin{tabular}{|c|c|c|c|c|}
\hline Rank & Gene symbol & Gene name & Function & Score \\
\hline 1 & $B E C N 1$ & Beclin 1 & Plays a central role in autophagy & $2.07 \mathrm{E}+20$ \\
\hline 2 & ATG7 & Autophagy-related gene 7 & $\begin{array}{l}\text { Associated with invasion, metastasis, and poor prognosis of several } \\
\text { cancers }\end{array}$ & $2.07 \mathrm{E}+20$ \\
\hline 3 & PIKЗC3 & $\begin{array}{l}\text { Phosphatidylinositol } 3 \text {-kinase } \\
\text { catalytic subunit type } 3\end{array}$ & Promotes the metastasis of colorectal cancer & $2.07 \mathrm{E}+20$ \\
\hline 4 & ATG12 & Autophagy related 12 & $\begin{array}{l}\text { Downregulation of ATG12 is required for survival of malignant intestinal } \\
\text { epithelial cells }\end{array}$ & $2.07 \mathrm{E}+20$ \\
\hline 5 & $G A B A R A P L 1$ & $\begin{array}{l}\text { GABA type A receptor } \\
\text { associated protein like } 1\end{array}$ & $\begin{array}{l}\text { Low expression is associated with a poor outcome in hepatocellular } \\
\text { carcinoma patients }\end{array}$ & $2.07 \mathrm{E}+20$ \\
\hline 6 & GABARAPL2 & $\begin{array}{l}\text { GABA type A receptor } \\
\text { associated protein like } 2\end{array}$ & Associated with proliferation and metastasis of colorectal cancer & $2.07 \mathrm{E}+20$ \\
\hline 7 & SQSTM1 & Sequestosome 1 & As a multifunctional hub participating in tumorigenesis & $2.07 \mathrm{E}+20$ \\
\hline 8 & $M A P 1 L C 3 A$ & $\begin{array}{l}\text { Microtubule associated protein } \\
1 \text { light chain } 3 \text { alpha }\end{array}$ & Related to microtubule binding and phosphatidylethanolamine binding & $2.07 \mathrm{E}+20$ \\
\hline 9 & $M A P 1 L C 3 B$ & $\begin{array}{l}\text { Microtubule associated protein } \\
1 \text { light chain } 3 \text { beta }\end{array}$ & Related to microtubule binding & $2.07 \mathrm{E}+20$ \\
\hline 10 & GABARAP & $\begin{array}{l}\text { GABA type A receptor- } \\
\text { associated protein }\end{array}$ & $\begin{array}{l}\text { Essential for the formation of autophagosomes during autophagy, and the } \\
\text { sequestration of cargo during selective autophagy }\end{array}$ & $2.07 \mathrm{E}+20$ \\
\hline
\end{tabular}

Table 2. Functions of genes in the prognostic autophagy-related gene signature

\begin{tabular}{|c|c|c|c|}
\hline No & $\begin{array}{l}\text { Gene } \\
\text { symbol }\end{array}$ & Full name & Function \\
\hline 1 & $B N I P 3$ & BCL2 interacting protein 3 & Associated with invasion, metastasis, and poor prognosis of several cancers \\
\hline 2 & $M Y C$ & Myelocytomatosis oncogene & Associated with invasion, metastasis, and poor prognosis of several cancers \\
\hline 3 & $B A G 1$ & Bcl-2-associated athanogene-1 & Biomarker for early breast cancer prognosis \\
\hline 4 & CALCOCO2 & $\begin{array}{l}\text { Calcium-binding and coiled-coil } \\
\text { domain } 2\end{array}$ & Mediating macroautophagy in innate immunity \\
\hline 5 & ATF4 & Activating transcription factor 4 & Potential biomarker for esophageal squamous cell carcinoma prognosis \\
\hline 6 & $A M B R A 1$ & $\begin{array}{l}\text { Autophagy and beclin } 1 \text { regulator } \\
1\end{array}$ & Associated with poor prognosis of several cancers \\
\hline 7 & $E G F R$ & Epidermal growth factor receptor & Associated with invasion, metastasis, and poor prognosis of several cancers \\
\hline 8 & $M A P K 1$ & Mitogen-activated protein kinase 1 & $\begin{array}{l}\text { High expression is associated with cell proliferation, invasion, and metastasis of } \\
\text { osteosarcoma cells }\end{array}$ \\
\hline 9 & $P E X 3$ & Peroxisomal biogenesis factor 3 & Associated with the invasion and metastasis of ovarian cancer cells \\
\hline
\end{tabular}


A

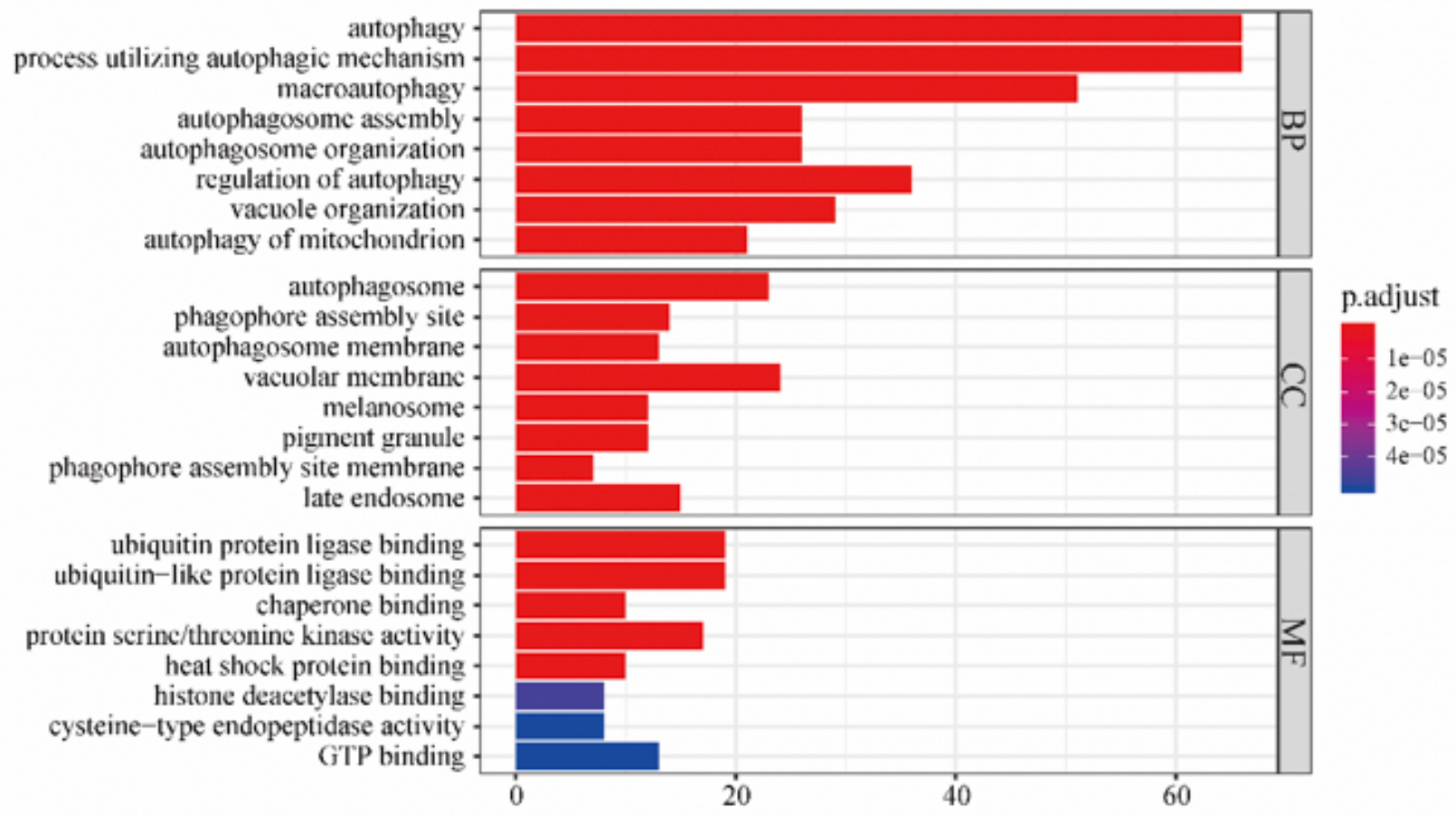

B

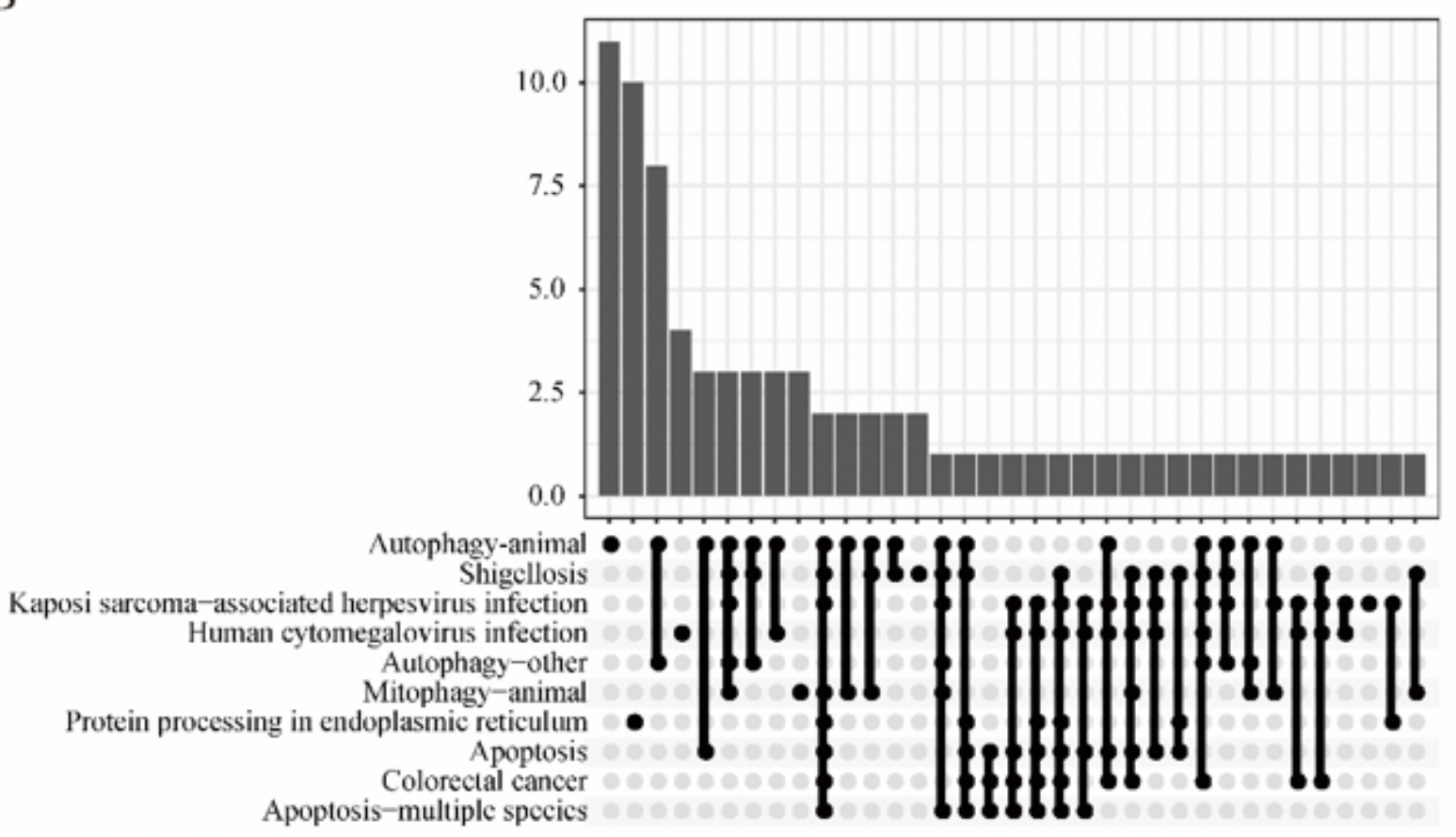

Figure 1

GO and KEGG pathway enrichment analysis. (A) GO biological function enrichment analysis. The color of the dot indicates the adjusted P-value: red, high; blue, low, and abscissas represent the number of autophagy-related genes; (B) KEGG pathway enrichment analysis. GO: gene ontology; KEGG: Kyoto Encyclopedia of Genes and Genomes; p.adjust: adjusted P-value; BP: biological process; CC: cellular component; MF: molecular function. 
A

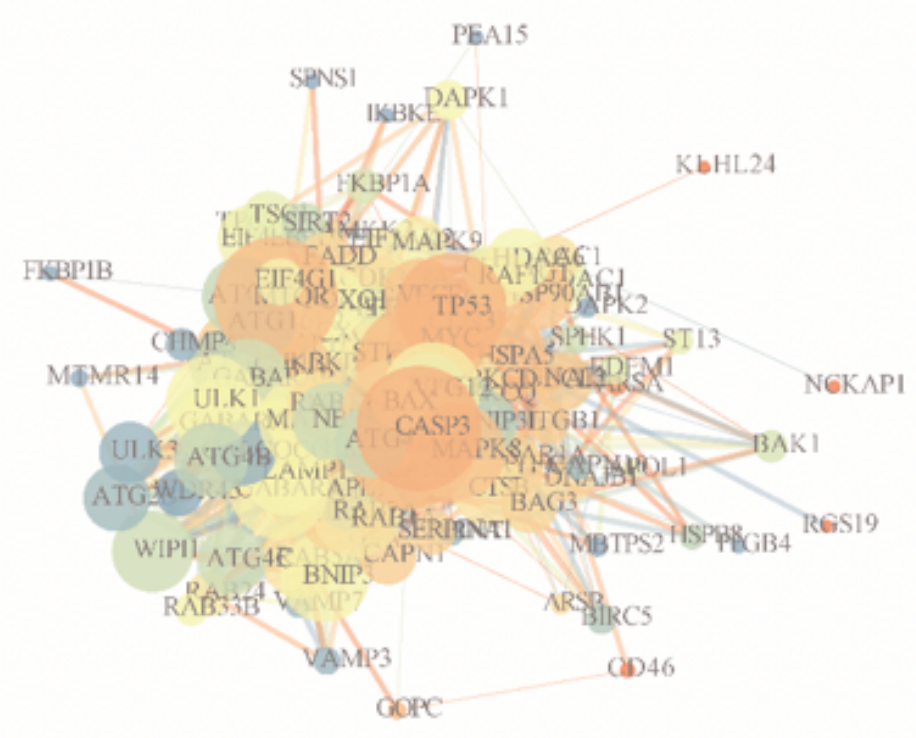

$\mathrm{B}$

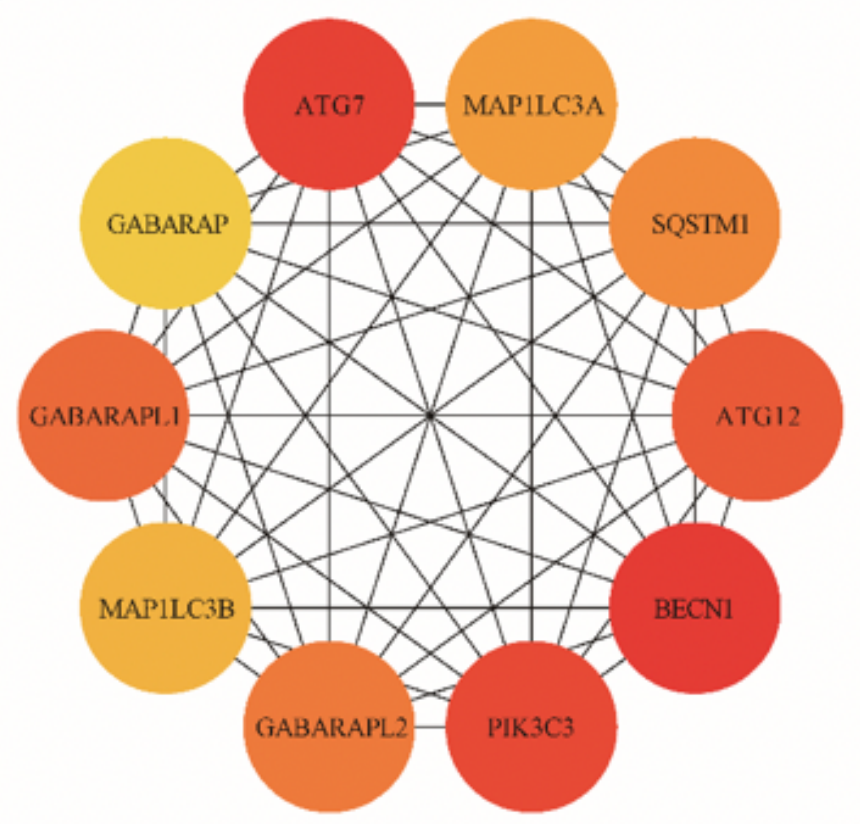

$\mathrm{C}$

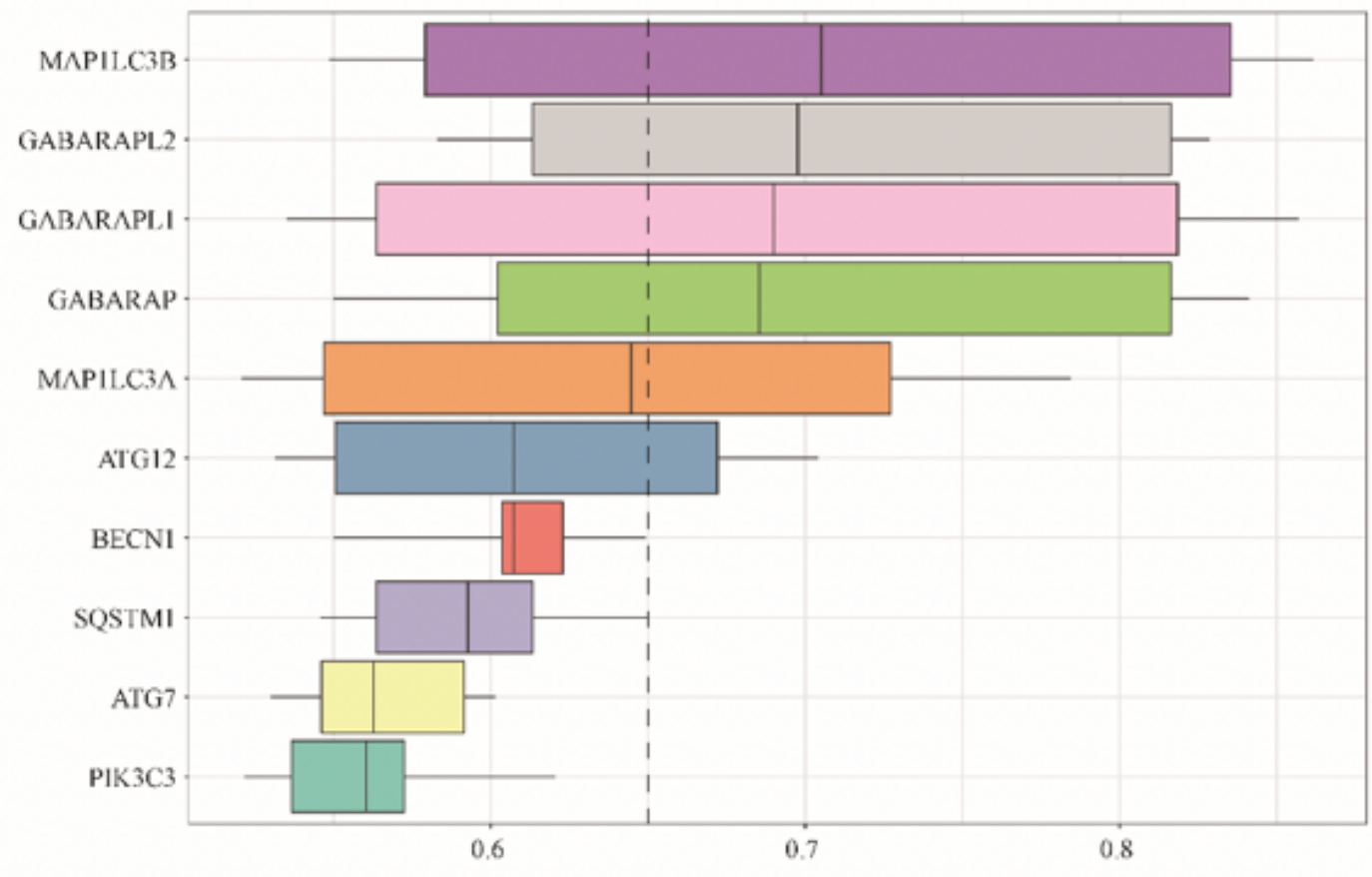

Figure 2

Analysis of PPI networks, hub autophagy-related genes, and their functional similarities. (A) PPI network analysis diagram; the node size indicates the clustering coefficient, the node color indicates the degree, the connection thickness indicates the comprehensive score, and the connection color indicates the coexpression. (B) The schematic diagram of the hub autophagy-related genes; the redder the color, the higher the enrichment score. (C) Functional similarity analysis of hub autophagy-related genes. The distribution of similar functions is summarized as a box chart. The boxes represent $50 \%$ similarity. The 
upper and lower boundaries show the 75th and 25th percentiles. The lines in the boxes represent the average value of functional similarity, which has a higher average. Genes with functional similarity (cutoff values> 0.7 ) are considered central genes in hub autophagy-related gene interactions, and the dashed lines indicate cut-off values.

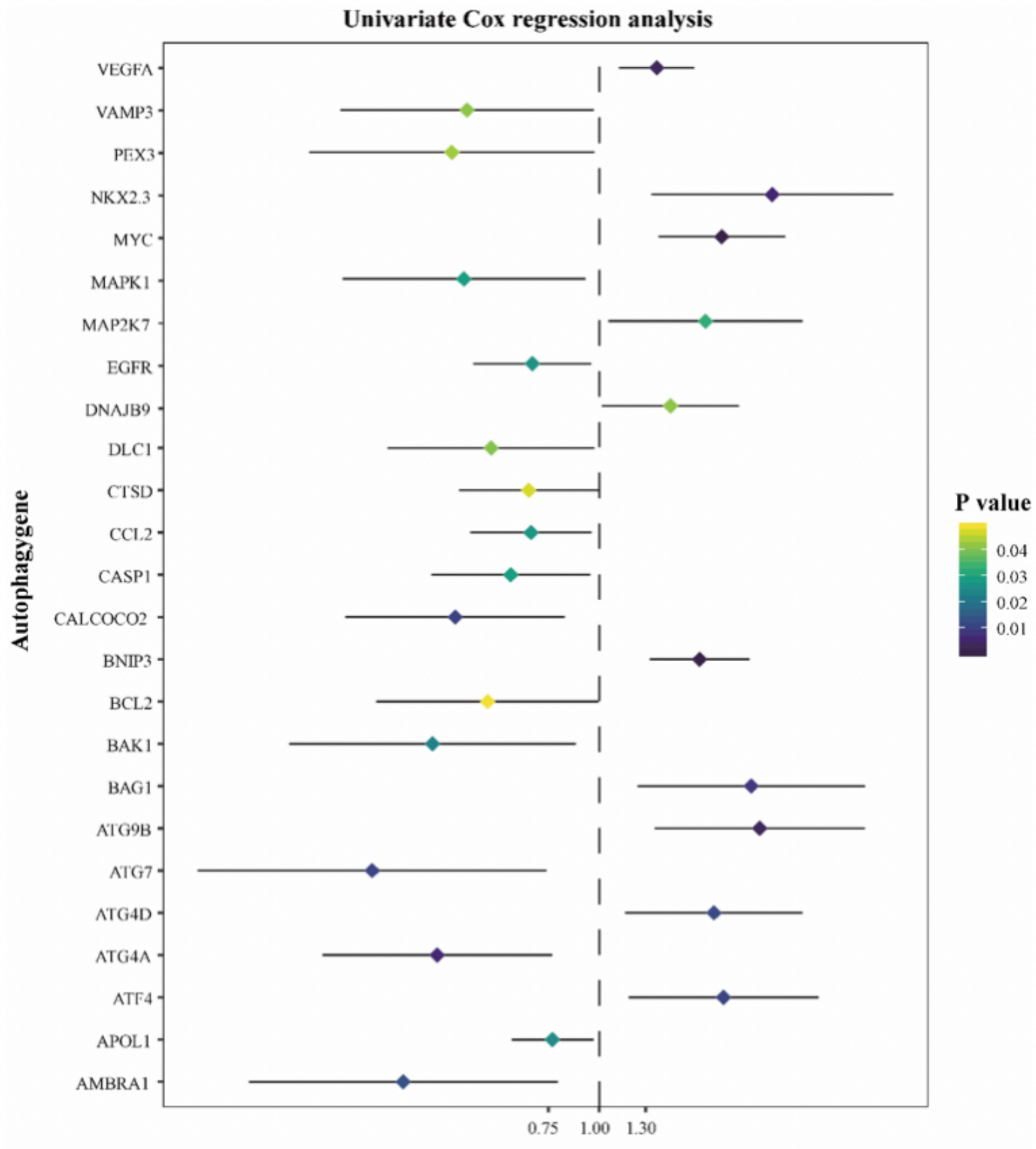

Hazard ratios of autophagy-related genes in osteosarcoma

Figure 3 
Univariate Cox regression analysis. The forest plot shows the top $25(25 / 120)$ significant prognostic autophagy-related genes. The more yellow the color, the larger the p-value; the bluer the color, the smaller the $p$-value.

A

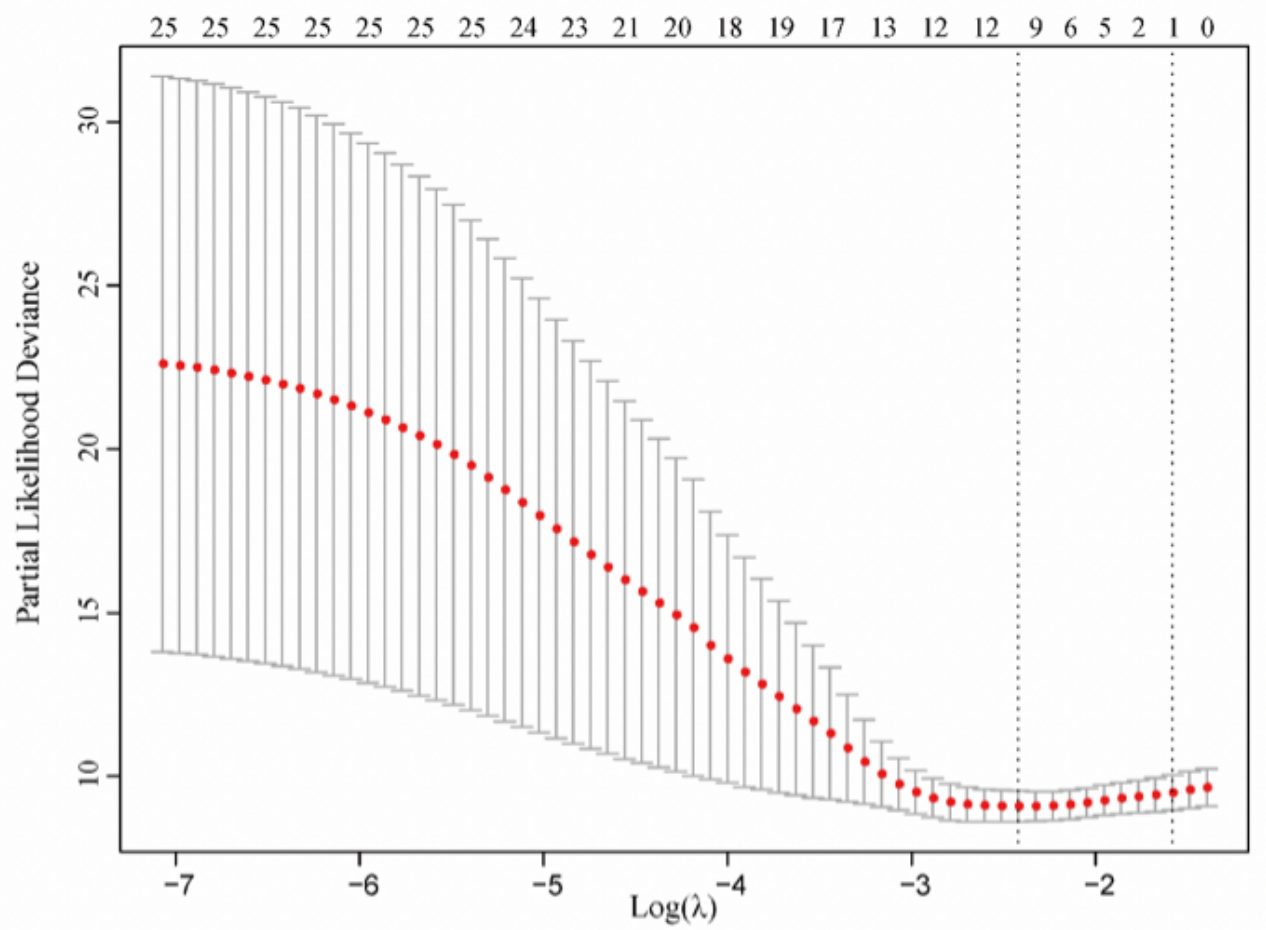

B

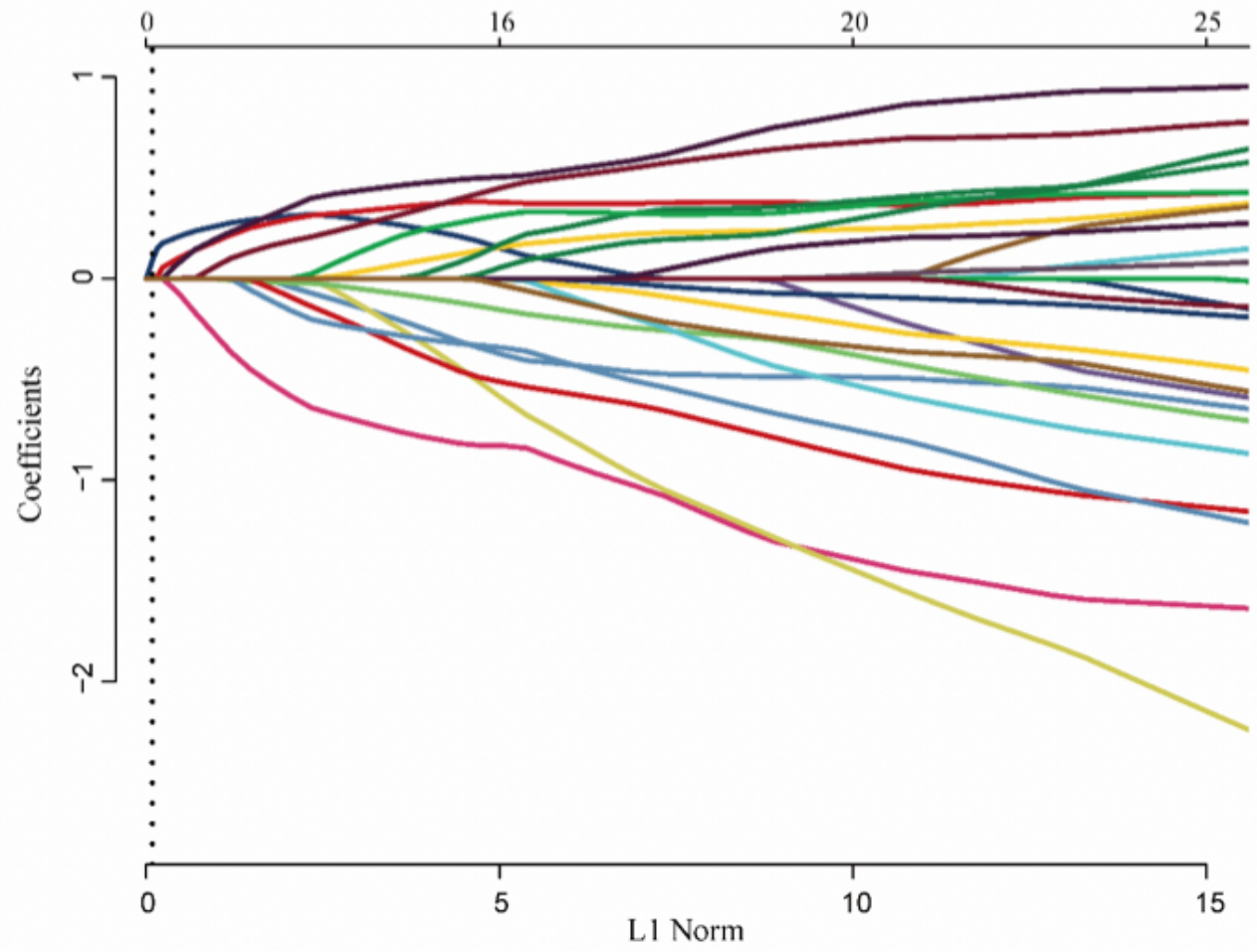

Figure 4

Lasso profiles of the 9 prognostic autophagy-related genes in OS. (A) Tuning parameter (lambda) selection in the LASSO model used 10-fold cross-validation via minimum criteria. (B) LASSO coefficient 
profiles of the prognostic autophagy-related genes.

A

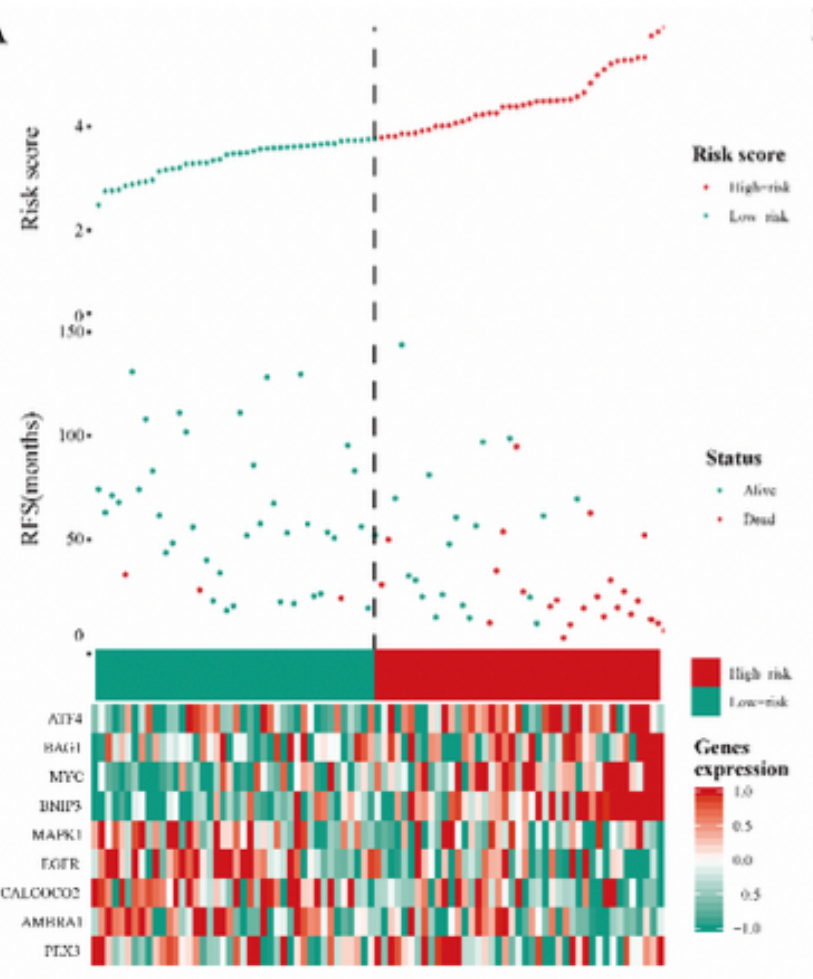

B

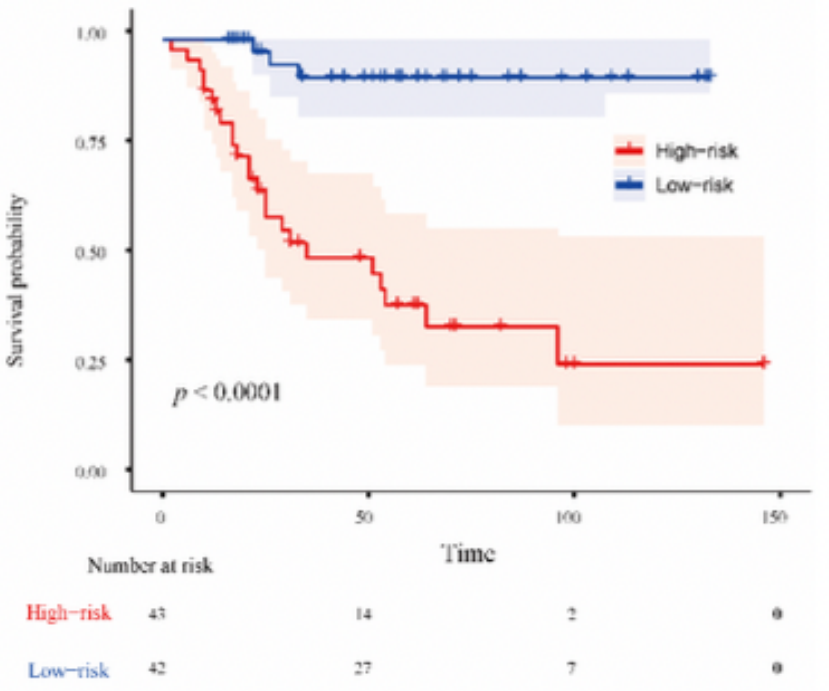

Figure 5

Characteristics of the prognostic autophagy-related genes signature (A) The distribution of prognostic autophagy-related genes risk score, patient survival status, and gene expression heatmap were analyzed. The black dotted line is the optimal cut-off value for dividing patients into low-risk and high-risk groups. (B) Kaplan-Meier survival curves for samples of the high-risk score group and the low-risk score group. The high-risk score is related to the overall poor survival of OS patients. The red line represents the high gene expression group, and the blue line represents the low gene expression group. $P<0.05$ is statistically significant. 
A

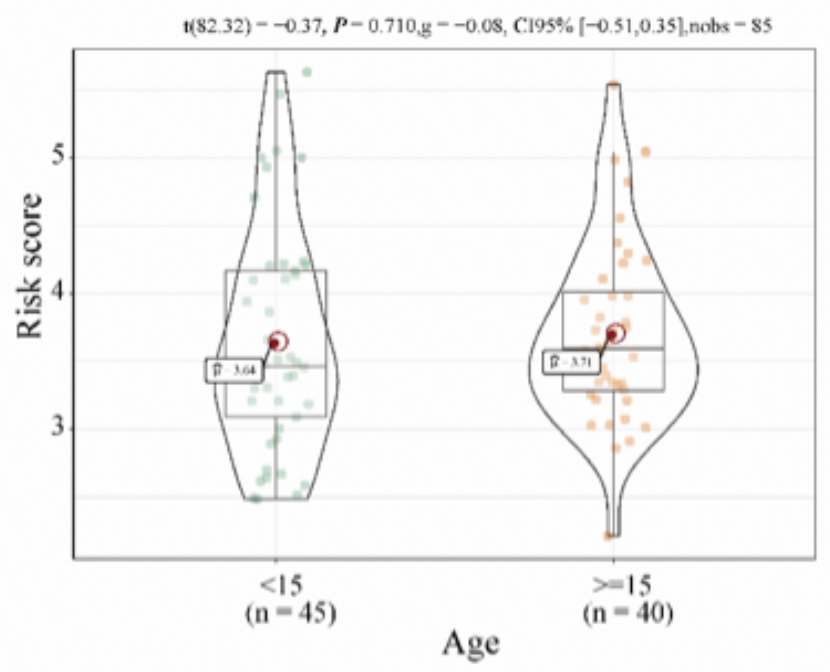

B

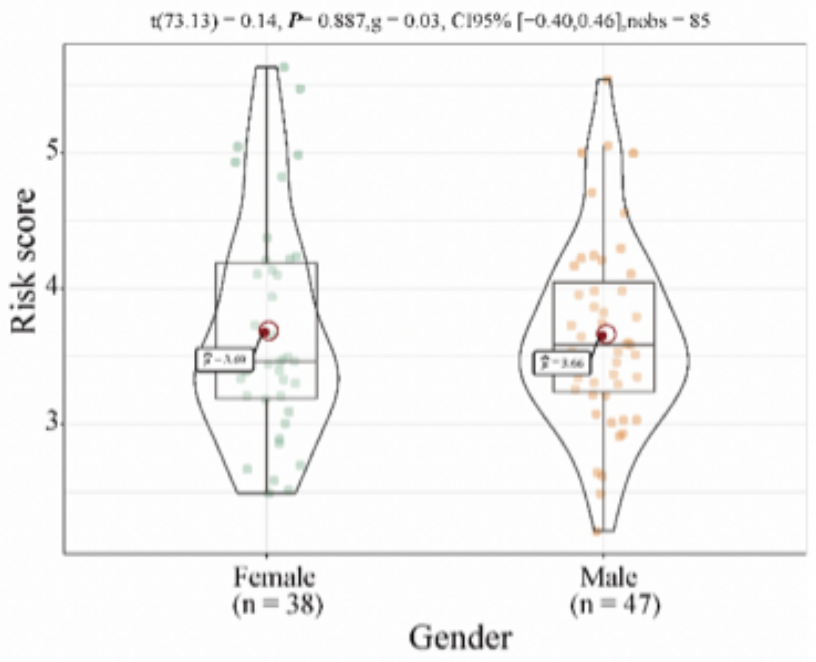

$\mathrm{C}$

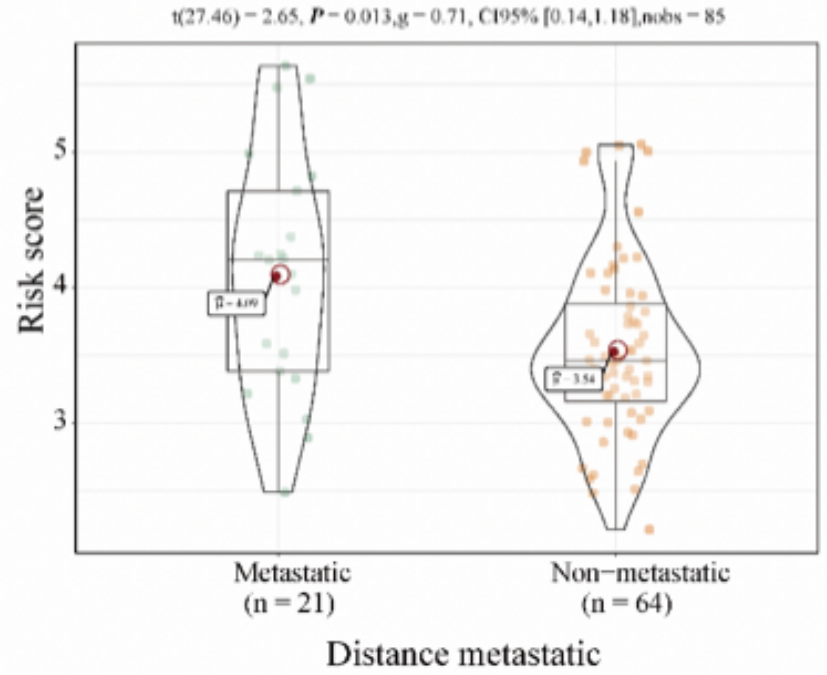

Figure 6

Correlation analysis between prognostic autophagy-related genes signature and clinical characteristics. (A) Correlation analysis of risk scores and age. (B) Correlation analysis of risk scores and gender. (C) Correlation analysis of risk scores and distant metastasis.

\section{Supplementary Files}

This is a list of supplementary files associated with this preprint. Click to download.

- Supplementarymaterial2.csv

- Supplementarymaterial1.csv 